\title{
Advances in Integrated Vehicle Health Monitoring Systems
}

\author{
$\underline{\text { doi:10.3991/ijim.v5i3.1671 }}$ \\ Debopam Acharya ${ }^{1}$ and Hyo-Joo Han ${ }^{2}$ \\ ${ }^{1}$ Computer Science, Georgia Southern University, GA U.S.A. \\ ${ }^{2}$ Information Systems, Georgia Southern University, GA U.S.A.
}

\begin{abstract}
One of the fastest growing fields of study in defense market currently is Integrated Vehicle Health Monitoring (IVHM). These systems perform collection and analysis of data concerning operating parameters and damage information of vehicles in real-time and periodically send them to the base station for appropriate action. Recent advances in materials and sensor networks and technologies promise development of such systems for land, water, and aerial vehicles. These IVHM systems are of immense use in defense services which require their vehicles and systems to operate normally even under hostile and harsh environments. This work will discuss various issues related to IVHMs and prominent sensor technologies available to build such systems. It will also include the structure of a general purpose IVHM of a vehicle which can be used to monitor its inner operating parameters and damage information of its components.
\end{abstract}

Index Terms-Integrated Vehicle Health Monitoring (IVHM), Sensor, Data Aggregation, Flat Routing, Hierarchical Routing

\section{INTRODUCTION}

Integrated vehicle health monitoring (IVHM) system is fast developing into a very active field of research and development. Although vehicle health monitoring is not a new concept, recent advances in the field of microelectronics, nanotechnology, sensors and sensing technologies have brought about a renewed interest in the field of IVHM. An IVHM facilitates collection and analysis of data concerning operating parameters and damage information of vehicles in real-time. This leads to an increase in vehicle safety conditions, decrease in maintenance cost and significant increase in operational life of the vehicle. Continuous operational monitoring helps manage the frequency and extent of maintenance procedures for various components within a vehicle. The development of real time localized inspection methods adds the ability to pinpoint damage in vehicles thus reducing or even eliminating the need for lengthy periodic inspections covering large areas of a vehicle. A long term vision would be to develop systems that would monitor and collect operational data, and after analyzing, self repairs the defective part leading to a reliable ageless system. IVHMs are a crucial part of defense vehicles as they operate in stringent atmospheric conditions. IVHMs in defense vehicles require greater initial investment during the development phase with the returns being greater capability and high utilization for a reduced total ownership cost over the service life of a fleet of vehicles. Hence, the purpose of IVHM is to provide a balance between maximizing the operational benefit and minimizing the development cost. Vehicle health monitoring systems are also getting popular in automobiles and are already present in some form in high end car market segment.

The successful operation of IVHM depends on a network of wired or wireless sensors, data aggregation sensor units, a central processing unit, and logistics and maintenance personnel (Figure 1). An important component of IVHM systems is the array of sensors. Sensors must be small, lightweight and networked to an onboard data aggregation sensor unit. They should also be capable of withstanding vehicle's operating conditions and environment, insusceptible to electromagnetic interference, both reliable and accurate and require low power input. One of the core functions of an IVHM is continuous data collection from a large number of sensors. This is done by data aggregation sensor units. Efficiently managing this high volume of data is central to the performance of an IVHM. Since the volume of data collected over a period of time can be very large, it may not be possible for the data aggregation sensor units to communicate all data to the central processing unit. Hence, it is the job of the data aggregation sensor units to generate relevant information from this high volume data before sending them to the central processing unit. An important constituent of data aggregation is the routing protocol running across the sensor network inside the vehicle. We will discuss few routing protocols that may be helpful in IVHMs.

\section{COUNTRIBUtion OF THIS WORK}

IVHMs have attracted attention from researchers all over the world. Yet, to the best of our knowledge there is no paper that summarizes functionalities, threats monitored in a vehicle and prominent sensors and technologies of a vehicle health monitoring system all in one place. In this paper, we have described all of these in detail. There are only few papers related to IVHMs and most of them are vehicle specific (fighter aircraft, spaceships, ground defense vehicles). To the best of our knowledge, there is no paper that describes the components and architecture of a general purpose IVHM. This is essential as any person intending to develop even a vehicle specific IVHM will need it in the initial phase of development. Hence, we developed and described a general purpose IVHM architecture that describes the basic components an IVHM must have.

\section{FUnCTIONALITY OF AN IVHM}

The key requirements of an IVHM are that it should be able to monitor and identify damaging events, its extent, seriousness, characteristics, and respond in an intelligent 
manner to mitigate the effects of the damage or propose its repair. Although a pure monitoring system is expected only to report damage, it is expected that the ultimate objective of an IVHM should also be able to formulate some kind of response to the damage.

The functionality of an IVHM [2, 3] can be subdivided as follows:

i. Detection of events: This requires complete information of the environment in which the vehicle will be operating, the threats it will face, use of appropriate sensors and a strategy for using them to detect events and respond within a given time frame.

ii. Characterization of the damage: This may be a sub-process of event detection. It may use same or different sensors (active or passive) depending upon the IVHM system at hand.

iii. Prioritization of the seriousness of the damage: An important component of an intelligent response is to identify the damages comprehensively and identify those damages that require immediate attention. In addition to that, the IVHM system should be able to determine the urgency (both relative and absolute) with which response to those damages should be formulated.

iv. Identification of the cause of the damage: An IVHM system should be able to collect data from a vast array of sensors seamlessly. Absence of any data (from a sensor) may be detrimental to formulate an accurate response.

v. Developing an effective response: Creating an effective and accurate response will depend on a number of factors such as the range of possible solution mechanisms, the nature and extent of the damage, availability of repair services, available response time, etc. A response normally consists of a sequence of actions. Major damage may demand an immediate repair or action such as complete isolation of a section of the vehicle, followed by a quick damage evaluation and repair strategy.

\section{THREATS MONITORED BY IVHMS}

An IVHM may need to monitor several types of threats. Threats to which a vehicle will be subject may be loosely divided between those produced directly by the environment (external threats), and those that arise from within the vehicle and its structure (internal threats). It is useful to distinctively identify various types of threats. The usefulness of making such a distinction is that external threats will depend strongly on the type of vehicle and its mission (e.g. aircraft, space shuttle, space vehicle, battle tanks, etc.), whereas the internal threats will be broadly similar for different vehicle types. The specific nature, severity and frequency of occurrence will depend on the materials, structure, and operating conditions of the vehicle.

The external threats to aerial vehicles [1] may include radiation from various sources (ionizing, non-ionizing, low and high energy, solar and cosmic, etc.), meteoroids and space debris, gravitation and upper atmospheric affects (atomic oxygen, plasma, etc.). External threats to land vehicles include ground materials, stringent atmospheric conditions, war zones, and so on. Internal threats are those generated within the vehicle, or indirectly due to the external environment. They include the following: i. Materials from which the vehicle is fabricated: This includes mechanical failure of materials (fracture, bond breaks, cracking, etc.), and degradation of material at surfaces and interfaces (wear, erosion, corrosion, adhesion, etc.).

ii. Fuel and propulsion systems: This may include engine failure (which may be due to material failure) or such things as fuel leaks or contamination.

iii. Energy and power systems: This may include generators (such as solar panels, nuclear, thermoelectric), batteries and storage devices, and the energy distribution system (wiring, insulation failures).

iv. Electronic systems: Guidance, Navigation and Control (GNC) systems, communications systems (internal and external), sensing and supervisory systems are some of the common electronic systems available in land and aerial vehicles.

v. Life support systems (where applicable): These may involve atmosphere (pressure, temperature, composition/contamination, explosive mixtures, etc.), water (quantity, purity, and contamination), food health systems (sensing, monitoring, and treatment).

Consequently it is clear that a comprehensive IVHM system will need to monitor broad range of measurement conditions and locations. Depending upon the vehicle, it may also have to measure a large variety of mechanical, electrical and chemical quantities, at a wide range of temporal and spatial scales.

\section{SEnsors And TECHNOLOGIES}

\section{A. Fiber Optic Sensors}

Sensors are indispensible in today's IVHMs. Wireless sensor networks and devices [8] are used to monitor environments to detect changes and relay back information in real-time to a server. Current IVHM systems are aimed at replacing traditional network cables with 802.15.4 wireless links between groups of sensors and aggregation systems [16]. IVHM systems using such intra-vehicle wireless transmission are suitable for long duration missions. Considering the large aggregation of vehicle structural elements, it is natural that extremely large numbers of sensors will be required for on-board structural integrity assessment. Fiber optic sensors have been identified as the leading candidate technology for meeting this requirement with a minimal weight penalty. Fiber optic sensors require a light source such as a laser, light emitting diode, or incandescent light. One of the main advantages of fiber optic sensors is that numerous sensor sites can be multiplexed along a single optical fiber, mitigating the complexity and weight inherent with the wiring required for a large number of single ended sensors. Fiber optic sensors can be used to measure strain, temperature, and pressure and also can be used for bond-line integrity, acoustic emission, and corrosion monitoring. Fiber optic sensors have several advantages: they are highly sensitive, may be surface mounted or embedded, and are insusceptible to electromagnetic interference. Multiple Bragg grating sensing systems, known as multiplexing, can be incorporated into a single fiber [7]. They are lightweight and can be used in a number of component geometries. Fibers must be handled carefully due to their brittleness. Commercial 
fiber optic sensors are currently available with research continuing into higher sensitivity sensors.

Fiber optic sensors designs can be of two types: cavity based designs and grating based designs [10, 17]. Cavity based designs utilize an interferometric cavity in the fiber to create the sensor. Examples include the extrinsic FabryPerot interferometer (EFPI) and the intrinsic or fiber Fabry-Perot interferometer (IFPI or FFPI). Although these designs have been incorporated in different applications such as in high temperature and electro mechanical impedance environments, they do not have multiplexing capability in a single fiber. Hence, they are not suited for applications requiring large number of sensors.

Grating based designs use a photo-induced or heatinduced periodicity in a fiber's core refractive index to create a sensor whose reflected or transmitted wavelength is a function of this periodicity. Grating based sensors (e.g., Bragg gratings) can be easily multiplexed by using gratings of different wavelength as in the case of wavelength division multiplexing (WDM). Factors limiting the number of sensors in a single fiber include limited bandwidth of the source as well as that supported by the fiber, and the range over which the physical parameter of interest is being measured.

\section{B. Piezoelectric Materials}

Piezoelectric materials [7] can be used for IVHM in several ways. Piezoelectric materials have the ability to convert a mechanical stress into an electrical signal thus acting like a sensor or convert an electric pulse into a mechanical strain (like an actuator). Piezoelectric sensors can detect energies emanating from impact events and defect generation, including crack formation and delamination. In this manner, arrays of sensors are used to monitor or detect such events. Each event has a unique energy signal associated with it, and through extensive data collecting, testing and field monitoring, real-time events can be identified and monitored. There is always a certain amount of background noise, hence significant experience and expertise is required to accurately diagnose the source.

Arrays of sensors also allow a pinpoint determination of an energy source's location. Sensors have been able to detect cracks 0.005 inches in length from a distance of six inches on flat plate samples. Sensors can be placed farther apart for more realistic crack sizes. The problem with this sensing method is that although crack and delaminations are readily detected, the exact size of the defect cannot be well-determined in the field. Crack and delamination lengths are critical parameters in determining maintenance of components. Piezoelectric sensors are passive devices requiring no energy input, which is also the case with fiber optics. They may be used on metals or composites, and may be surface mounted (patches) or embedded. A second potential use for piezoelectric material is active defect detection. This method is currently under research and development, particularly for use with composite materials. Here, an electric pulse is sent to a piezoelectric actuator which produces Lamb waves within a structure. An array of piezoelectric sensors will pick up the resultant Lamb waves for processing. If a defect such as delamination or corrosion exists within the array of sensors, a change in the signal results, which is distinct from the original non-defect component. These systems are calibrated by recording the signals in a structure before it is placed in service. The sensors will be able to locate the position of the defect within the structure, and in this case, the defect size can be determined from the degree of signal change.

\section{Active and Passive Ultrasonic Sensing}

Ultrasonic sensing, applied in both active and passive modes, is another sensor technology area receiving considerable attention. Evaluation of transmitted active ultrasonic signals is a methodology that has been used to identify and assess damage [17]. However, such approaches use sensors that are scanned over the structure to provide a point-by-point representation of material properties and/or damage locations. Such scanning probe approaches are not currently feasible for continuous onboard monitoring. Therefore, the use of arrays of permanently attached or embedded ultrasonic transducers, which act both as transmitters and receivers, is being researched. Ultrasonic signals generated by one transducer are detected by neighboring transducers within an array. Damage between the transducers can be identified, and with advanced analysis methods, material along propagation paths that include reflections from structural boundaries can also be tested. The development of the Stanford Multi-Actuator Receiver Transduction (SMART) layer is an excellent example of recent efforts in this area [11]. Ongoing areas of research in active ultrasonic sensing technology for structural health monitoring include 1) the further improvement and characterization of miniaturized, rugged, embeddable sensors, 2) analysis methodologies for optimized sensor placement to enable characterization of damage throughout the entire structure rather than just along direct propagation paths, and 3) ultrasonic wave propagation modeling that occurs when these sensors are attached or embedded on thin-walled aerospace structures.

Passive ultrasonic monitoring, also known as acoustic emission (AE), utilizes an array of ultrasonic sensors. The sensor array is used to passively monitor acoustic signals generated by damage mechanisms such as crack growth. $\mathrm{AE}$ is widely used as a conventional method for off-line structural assessment, and can also be implemented to monitor a structure while in service. This capability makes it well suited for onboard structural health monitoring of aerospace vehicles. However, considerably more research and development is being undertaken to make AE a more viable technology for IVHM. Successful implementation of $\mathrm{AE}$ requires sensors having lighter weight, increased sensitivity, and increased ruggedness over those currently available. Additionally, reductions in size, weight, and power requirements of the associated $\mathrm{AE}$ monitoring instrumentation are also needed. Modern $\mathrm{AE}$ analysis methodologies are not only expected to locate and identify damage accurately, but also discriminate extraneous noise from signals indicating actual damage. Current efforts in this research area include development of AE multiplexing instrumentation systems that can miniaturize AE flight systems, the development of fiber optic AE sensors [12] and the development of Modal AE based analysis methods [13]. Another significant development is that of modeling approaches to better understand and predict AE propagation phenomena [14]. Such models are advantageous for a number of reasons and include characterization of $\mathrm{AE}$ transducers, optimization of sensor placement on a structure, and development of new and automated $\mathrm{AE}$ data analysis methods. 


\section{Microelectromechanical devices}

Microelectromechanical devices (MEMs) incorporate an existing sensing technology, or multiple technologies into one device. Sensing technologies that are being transitioned to MEMs devices include strain gages, accelerometers, temperature sensors, humidity sensors, and corrosion sensors. In addition, wireless MEMs devices using radio frequency communications have been implemented. Such devices typically use a radio frequency identification chip (RFID) which is activated through a local processing unit. The RFID chip responds to the processing unit with a signal identifying the sensor as well as information from the sensing mechanisms. One downfall of MEMs devices is their susceptibility to electromagnetic interference.

\section{E. Wireless Remote Sensor Systems}

Several conventional sensors such as strain gages, thermocouples, and accelerometers are used for structural health monitoring. One major issue for such sensors is the need to route large numbers of wires to provide power and data communication. This is a difficult problem when retrofitting these sensors into existing structures, such as the aging aircraft fleet. To address this concern, a prototype adaptable vehicle health monitoring architecture has been developed [15] and flight tested. The architecture is self-contained and requires limited integration intrusion into existing systems. There are three operational levels to the architecture: one or more remote data acquisition units (RDAU) located throughout the vehicle; a command and control unit (CCU) located within the vehicle; and, a terminal collection unit (TCU) to collect analysis results from all vehicles.

The RDAUs are multi-sensor interfaces with an onboard miniature computer, programmable digital interface, nonvolatile solid-state memory and a wireless transceiver for communication with the command and control unit. Communication is achieved by using wireless radio frequency transceivers operating at $433 \mathrm{MHz}$. The RDAUs were designed to withstand impact during aircraft landing while mounted on the main landing gear, and have been vibration tested up to acceleration amplitude of $20 \mathrm{~g}$ at $2000 \mathrm{~Hz}$. It was also designed to operate in nonenvironmentally controlled locations of the plane. The RDAU was thermally tested for temperatures ranging from $-50^{\circ} \mathrm{C}$ to $55^{\circ} \mathrm{C}$ and pressure tested to simulate 50,000 $\mathrm{ft}$ altitude. Vibration tests verified that the remote data acquisition unit could operate at vibration levels representative of those experienced by commercial aircraft. During vibration testing, the final acceleration amplitude was $20 \mathrm{~g}$ at $2000 \mathrm{~Hz}$. The RDAU has a multiple channel programmable digital interface that allows user discretion in choosing type and number of sensors, sampling rate and sampling duration for each sensor chosen. Programmable data acquisition circuitry and expert systems trained to performance baselines in each RDAU allow the architecture to be adaptable for many types of vehicles and structures. Once a suite of sensors has been chosen for each RDAU and installed on the vehicle, a baseline of acceptable vehicle performance is established from measurements acquired when the vehicle is performing correctly. Each RDAU uses an embedded expert system trained to its respective baseline. The CCU is a computer-based subsystem that provides the communications, analysis repository, and user interface functions for the RDAUs. The CCU

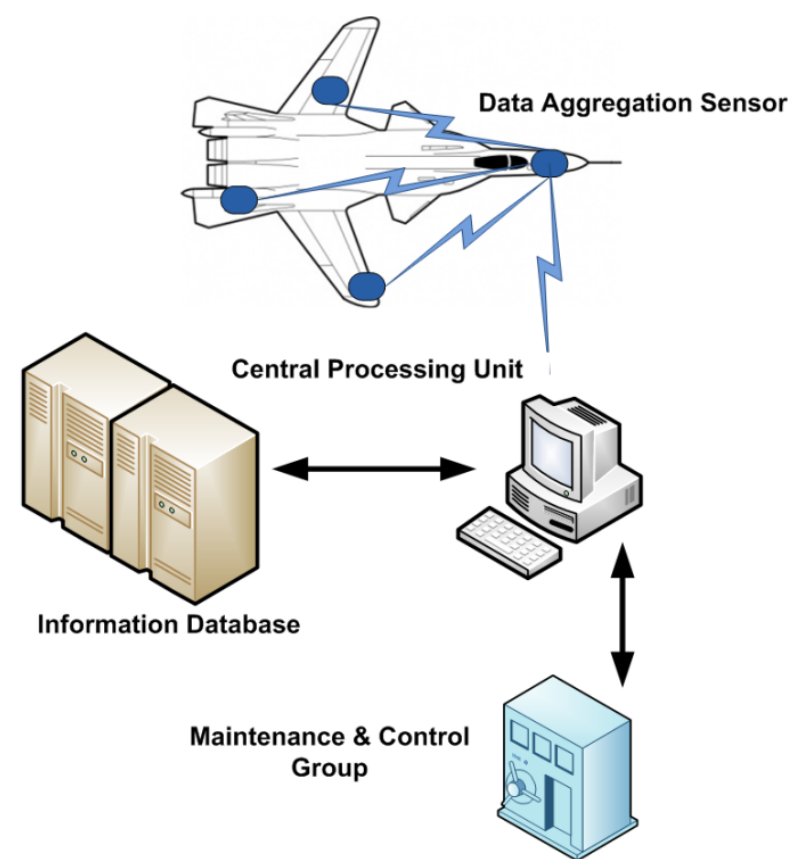

Figure 1. General Purpose Architecture of IVHM

can also serve as a power management tool by regulating when individual or combinations of RDAUs are powered. A radio frequency (RF) wireless network of RDAUs can be controlled from a single CCU. The TCU provides the means to autonomously retrieve vehicle analysis results from all vehicle CCUs. The TCU performs analysis on results collected from all vehicles to identify any fleetwide anomalies (e.g., all aircraft have the same faulty bearing at a similar location). The TCU develops the final summary of the vehicle health monitoring results that gets routed to the appropriate users (e.g., maintenance workers, airlines operations, etc.).

\section{Architecture of A Vehicle Health Monitoring SYSTEM}

A general purpose architecture of vehicle health monitoring system is given in Figure 1. Minor changes should be made to portions the architecture to suit vehicles in different terrains and conditions (land, air, water, civilian, military, etc.). Each individual component of the architecture is described below:

\section{A. Array of Sensors}

The architecture consists of an array of sensors in the vehicle. Sensor nodes [9] are hardware devices that produce measurable response to a change in physical conditions like temperature, pressure, humidity, acceleration, light intensity, etc. Functions of a sensor include sensing or measuring physical data of the area to be monitored. Signals sent from sensors either reach the destination directly or have to be aggregated by another intermediate sensor node. These data aggregation sensor nodes then transfer data to the server for further processing. Aggregation sensor node may also need to filter out unnecessary or redundant data and send only useful information to the server. Sensor nodes should be of small size, should consume extremely low energy, operate autonomous and unattended, and always stay adaptive to the environment. As wireless sensor nodes are micro-electronic sensor device, they can only be equipped with a limited power 
source. In an IVHM, sensor nodes will monitor operational (both structural and technical) conditions in the vehicle. The sensors send this maintenance log regularly to a data aggregation sensor. Various types of sensors may be fitted to a vehicle depending upon its function and operation. Sensor technologies used in IVHMs have been extensively discussed in section 4 .

\section{B. Sensor Data Aggregation}

Data aggregation in sensor network is a widely discussed topic $[4,6]$. When an event occurs, sensors gather data and communicate it to the end sensor node. As the number of nodes in the sensor network increases the probability of congestion also increases. This congestion leads to sub-optimal routing performance. Additionally, lots of packets get dropped and they need to be retransmitted again. Hence, the over-all response time increases. Data aggregation is a technique which tries to alleviate this congestion problem. It attempts to collect useful information from the sensors surrounding the event. It then transmits only the useful information to the end point thereby reducing congestion and its associated problems. In our architecture, data aggregation sensors have an important job of selectively aggregating useful information and transfer it to the vehicle monitoring center using wireless communication. It is also responsible for discarding any unnecessary data as it may lead to redundant data transfer to the monitoring station. Hence, only useful information rather than unnecessary huge volume of data will be transferred to the central processing unit. Routing protocols are the backbones of data aggregation schemes. We discuss two broad types of routing protocols in this chapter. Depending upon the conditions (type of vehicle, rate of data transfer, operating conditions, etc.) one or more of the schemes may be used.

\section{1) Flat Routing}

The first category of routing protocols is the multi-hop flat routing protocols. In flat routing based networks, each node typically plays the same role and sensor nodes collaborate to perform the sensing task. Presence of large number of identical nodes makes it infeasible to assign a global identifier to each node. This consideration has led to data-centric routing, where the data aggregation node sends queries to certain regions and waits for data from the sensors located in the selected regions. Data is normally requested using queries, and hence attribute based naming is necessary to specify the properties of data.

One of the popular types of flat routing protocols has been proposed by Heinzelman et al $[4,5]$. They proposed a family of adaptive routing protocols called Sensor Protocols for Information via Negotiation (SPIN) that disseminate all the information at each node to every node in the network assuming all nodes as potential aggregation nodes. This allows a user to query any node and get the required information immediately. These protocols make use of the property that nodes in close proximity have similar data, and hence there is a need to only distribute data other nodes do not posses. The SPIN family of protocols uses data negotiation and resource-adaptive algorithms. Nodes running SPIN assign a high-level name to completely describe their collected data (called meta-data) and perform metadata negotiations before any data is transmitted. This ensures that there is no redundant data sent throughout the network. In addition, SPIN has access to current energy levels of the nodes and adapts the proto- col it is running based on how much energy remains at these nodes. These protocols work seamlessly and broadcast information all over the network, even when a request to access data has not been made.

\section{2) Hierarchical Routing}

Hierarchical based routing methods are popular techniques that have advantages like scalability and efficient communication. Hierarchical routing is also utilized to perform energy-efficient routing in sensor networks as they are normally power constrained. In a hierarchical routing architecture, higher energy nodes can be used to aggregate and send information to the server, while low energy nodes can be used just to perform the sensing the target. This concept of creation of clusters where special tasks are assigned to cluster heads can greatly contribute to overall system scalability, lifetime, and energy efficiency. Hierarchical routing is an efficient method to lower energy consumption within a cluster as it leads to a net decrease in the total number of transmitted messages to the data aggregation node. Heinzelman et al $[4,5]$ introduced a hierarchical clustering protocol for sensor networks, called Low Energy Adaptive Clustering Hierarchy (LEACH). LEACH is a cluster-based protocol, which includes distributed cluster formation. LEACH randomly selects few sensor nodes as cluster heads (CHs) and rotates this role to evenly distribute the energy load among the sensors in the network. In $\mathrm{LEACH}$, the $\mathrm{CH}$ nodes compress data arriving from nodes that belong to the respective cluster, and send an aggregated packet to the data aggregation node (in order to reduce the amount of information that must be transmitted). LEACH uses a TDMA/code-division multiple access (CDMA) MAC to reduce inter-cluster and intra-cluster collisions. Therefore, this protocol is most appropriate when there is a need for constant monitoring by the sensor network. Since this is the basic purpose of an IVHM, data aggregation node in such systems should use hierarchical protocols.

\section{Central Processing Unit}

The data aggregation sensor collects useful information and passes it to the central processing unit. The central processor has the important job of processing and analyzing incoming data. Suitable test cases in the central processing unit will use incoming information (from the data aggregation sensor) to generate results. These results will be compared with the simulation test results generated earlier during lab testing of equipments. The report generated will depict the current operation status of each component. All the reports should be organized and send to an information database. Maintenance and control personnel should be able to access the database anytime, anywhere.

\section{Maintenance and Control Group}

In the base station, the Maintenance and Control Group (MCG) support post-processing of data. Their activity includes usage trending and health prognostic functions that require vehicle and fleet historical data. The MCG collates historical condition, usage, maintenance, and diagnostic/prognostic data with current report (generated by the central processing unit) to produce accurate and optimum diagnostic and/or prognostic data for the maintenance personnel. This critical process of health assessment may incorporate Bayesian network models to describe failure probabilities of various subsystems. The report 
generated helps the maintenance personnel in deciding what to repair and when to perform the repair as a function of operational requirements and resource availability. The report also helps the personnel in generating an estimate of remaining useful life of a component or subsystem for given usage profiles.

\section{CONCLUSION}

Recent technology advances in wireless sensor networks, developments in technologies such as MEMS, microelectronics, nanotechnology and wireless communication networks have lead to the development of integrated vehicle health monitoring systems. These systems are now an important component in most military vehicles on land, sea and air. The chapter discusses functionalities expected from an IVHM and identifies threats which an IVHM can monitor. In the longer term, these systems will provide a basis for the development of self-repairing, and perhaps even ageless, structures. In the shorter term, IVHM could allow more efficient design leading to the decrease in overall cost of redundant simulation and testing, and reduce maintenance cost and inspection requirements. All these will ultimately make the operating environment more secure for the passengers inside the vehicle.

\section{REFERENCES}

[1] Abbott, D., Doyle, B., Dunlop, J. B., Farmer, A. J., Hedley, M., Herrmann, J., James, G. C., Johnson, M. E., Joshi, B., Poulton, G. T., Price, D. C., Prokopenko, M., Reda, T., Rees, D. E., Scott, D. A., Valencia, P., Ward, D., Winter, J. G.: Concepts for an Integrated Vehicle Health Monitoring System. In: Review of Progress in Quantitative Nondestructive Evaluation, Vol. 22, pp. 1606-14 (eds. D.O. Thompson and D.E. Chimenti), American Institute of Physics Conference Proceedings ,Vol. 657, pp. 1606-1614, 2003..

[2] Price, D., Scott, A., Edwards, G., Batten, A., Farmer, A., , Johnson, M., Lewis, C., Poulton, G., Prokopenko, M., Valencia, P., Wang, P.: An Integrated Health Monitoring System for an Ageless Aerospace Vehicle. In: 4th International Workshop on Structural Health Monitoring, pp. 310-318, September 2003, Stanford, CA, USA

[3] Price, D., Batten, A., Edwards, G., A., Farmer, Gerasimov, V., Hedley, M., Hoschke, N., Johnson, M., Lewis, C, Murdoch, A., Prokopenko, M., Scott, A., Valencia, P., Wang. P.: Detection, Evaluation and Diagnosis of Impact Damage in a Complex MultiAgent Structural Health Management System. In: 2nd Australasian Workshop on Structural Health Monitoring, pp 16-27, December 2004, Melbourne, Australia.

[4] Heinzelman, W., Chandrakasan, A., Balakrishnan, H.: EnergyEfficient Communication Protocol for Wireless Microsensor Networks. In: 33rd Hawaii International Conference on Systems Sciences, pp 1-10, January 2000, Hawaii, USA. doi:10.1109/ HICSS.2000.926982

[5] Heinzelman, W., Kulik, J., Balakrishnan, H.: Adaptive Protocols for Information Dissemination in Wireless Sensor Networks. In: 5th ACM Annual International Conference on Mobile Computing and Networking, pp. 174-85, August 1999, Seattle, WA, USA.

[6] Kulik, J., Heinzelman, W., Balakrishnan, H.: Negotiation-Based Protocols for Disseminating Information in Wireless Sensor Net- works. Wireless Networks. 8, 169--85 (2002). doi:10.1023/ A:1013715909417

[7] Advanced Materials, Manufacturing and Testing Information Analysis Center, http://ammtiac.alionscience.com/pdf/2004MaterialEASE27.pdf

[8] Crossbow Technology: Low-Power Wireless Mote Solutions Overview,

http://www.xbow.com/Products/wproductsoverview.aspx

[9] Crossbow Technology: Wireless, http://www.xbow.com/Home/wHomePage.aspx

[10] Steenkiste, R. J. and Springer, G. S., Strain and Tempertaure Measurement with Fiber Optic Sensors, Technomic Publishing (Lancaster, PA), pp. 3-4 (1997).

[11] M. Lin, 2nd International Workshop on Structural Health Monitoring, Stanford University Technomic, pp. 603-611 (1999).

[12] Duke, J. C., Cassino, C. D., Childers, B. A., and Prosser, W. H.: Characterization of an EFPI AE Sensor, submitted to Research in Nondestructive Evaluation (2002).

[13] Gorman, M.R, Progress in QNDE, Vol. 17, eds. D. O. Thompson and D.E. Chimenti, Plenum Press, New York, pp. 557-564 (1998).

[14] Prosser, W. H., Hamstad, M. A, Gary, J, and Gallagher, A.O. Reflections of AE Waves in Finite Plates; Finite Element Modeling and Experimental Measurements, Journal of Acoustic Emission, Vol. 17(1-2), pp. 37-47 (1999).

[15] Woodard, S. E., Coffey, N. c., Taylor, B. D., Brett, R. R. and Woodman, K. L.: Autonomous Health Monitoring Architecture and Hardware, United States Patent Provisional Application and NASA Langley Research Center Invention Disclosure LAR 16407-1, Feb 2002.

[16] Prosser, W. H., Brown, T. L., Woodard, S.E., Fleming, G. A. and Cooper, E.G.: Sensor Technology for Integrated Vehicle Health Management of Aerospace Vehicles, in 29th Annual Review of Progress in Quantitative Nondestructive Evaluation (2003).

[17] Prosser, W. H., Allison, S. G., Woodard, S. E., Wincheski, R. A., Cooper, E. G., Price, D. C., Hedley, M., Prokopenko, M., Scott, D. A., Tessler A. and Spangler, J. L.: Structural Health Management for Future Aerospace Vehicles, in 2nd Australasian Workshop on Structural Health Monitoring, Melbourne, Australia, 2004.

\section{AUTHORS}

Debopam Acharya (dacharya@georgiasouthern.edu) is an Assistant Professor of Computer Sciences at Georgia Southern University at Statesboro, GA. He holds a PhD from University of Missouri. He is a member of IEEE. His research interests include Context Aware Computing, Mobile Healthcare and Social Network Analysis.

Hyo-Joo Han (hhan@georgiasouthern.edu) is an Associate Professor of Information Systems at Georgia Southern University. She holds a PhD from New Jersey Institute of Technology. Her research interests include Pervasive Computing, Computer-Mediated Communication, Computer-Supported Cooperative Work, Education in I.T., and E-Commerce.

This article is an extended version of a paper presented at the Interdisciplinary Conference of AHLiST 2010 Conference, June 2010, Madrid, Spain. Received May $5^{\text {th }}, 2011$. Published as resubmitted by the authors June $9^{\text {th }}, 2011$. 